Article

\title{
Flocculation of a High-Turbidity Kaolin Suspension Using Hydrophobic Modified Quaternary Ammonium Salt Polyacrylamide
}

\author{
Jiangya Ma ${ }^{1,2, * \mathbb{C}}$, Xue Fu ${ }^{1}$, Wei Xia ${ }^{1}$, Kun Fu ${ }^{3}$ and Yi Liao ${ }^{4}$ \\ 1 School of Civil Engineering and Architecture, Anhui University of Technology, Maanshan 243002, China; \\ ahutmunicipal@126.com (X.F.); bylch2008@sina.com (W.X.) \\ 2 Engineering Research Center of Biomembrane Water Purification and Utilization Technology, \\ Ministry of Education, Maanshan 243002, China \\ 3 Key Laboratory of Yangtze River Water Environment, Ministry of Education, Tongji University, \\ Shanghai 200092, China; fukun@tongji.edu.cn \\ 4 Department of Civil Engineering, Sichuan University of Science and Engineering, Zigong, 643000, China; \\ ly-001-1980@163.com \\ * Correspondence: majiang_ya@126.com
}

Received: 25 January 2019; Accepted: 12 February 2019; Published: 18 February 2019

\begin{abstract}
In this work, a novel cationic polyacrylamide (PAMD) was synthesized by acrylamide (AM) diallyl dimethyl ammonium chloride (DMD) and dodecyl polyglucoside (DPL) under low-pressure ultraviolet (UV) initiation. The intrinsic viscosity and cationic degree of PAMD were optimized in copolymerization. The optimum synthesis conditions that affect polymerization were determined to be solid content $30 \%$, DPL content $25 \%$, DMD content $30 \%$, illumination time $135 \mathrm{~min}$, and pH 9 . The flocculation performance of flocculant PAMD with a high cationic degree was investigated in the purification of high-turbidity water. The flocculation mechanism was correspondingly studied and summarized based on Fourier transform-infrared (FTIR) analysis. Finally, the results of an experimental simulation using the response surface method show that $98.9 \%$ supernatant transmittance was achieved under dosage $4 \mathrm{mg} / \mathrm{L}$, fast stirring time $20 \mathrm{~min}, \mathrm{pH} 7$, and stirring speed $320 \mathrm{rpm}$.
\end{abstract}

Keywords: high turbidity; kaolin; hydrophobic; quaternary ammonium salt; polyacrylamide

\section{Introduction}

Water treatment has always been a major problem in environmental governance. The purification of high-turbidity water caused by industrialization and urbanization is one of the serious problems in water treatment $[1,2]$. Various technologies, such as adsorption, flocculation, electrochemical oxidation, and biotechnology, have been researched and applied in water treatment [3-5]. Among them, flocculation is cost-effective and convenient [6]. In the flocculation process, the flocculation efficiency mainly depends on the selected flocculants.

Flocculants are divided into two categories according to the different materials: one is inorganic coagulants, such as aluminum sulfate, poly (ferric chloride) and poly (aluminum chloride) [7], which have been used in high-turbidity water purification. However, the application of inorganic coagulants in high-turbidity water treatment is restricted by the excessive amount needed, the uneconomical cost, and residual chemicals [8]. The other is organic polymer flocculants, such as polyacrylamide and its derivatives, which have been developed and widely used due to their satisfactory solid-water separation performance [9]. Considering the toxicity of acrylamide (AM) and the charge neutralization effect for aggregation of pollutant particles, a cationic monomer was used in 
synthesis of polyacrylamide [10,11]. Generally, the extensively used cationic polyacrylamide(CPAM) is produced by copolymerization of AM and cationic monomers. The commonly used cationic monomers are methacryloxy ethyl trimethyl ammonium chloride (DMC), [2-(acryloyloxy) ethyl]-trimethyl ammonium chloride (AOTAC), and diallyl dimethyl ammonium chloride (DMD) [12-14]. DMD is unanimously approved because of its molecule containing two double bonds [15]. A cationic polyacrylamide flocculant synthesized by DMD, AM, and hydrophobic monomer butylacrylate (BA) was reported to have its flocculation performance obviously improved due to hydrophobic associations [16]. However, a hydrophobic monomer that does not easily dissolve in solution has a bad influence on the synthesis effect [16]. In this study, dodecyl polyglucoside (DPL) was selected because of its satisfactory solubility.

At present, commonly used initiation polymerization methods have been reported, including thermal initiation polymerization, radiation initiation polymerization, microwave initiation polymerization, and photo-initiation polymerization [17-19]. Among them, the ultraviolet (UV) initiation method, because it is environmentally friendly and has a rapid reaction rate, has been widely adopted by researchers at home and abroad. Zheng et al. [20] reported that the anionic polyacrylamide P (AM/AA/AMPS) was synthesized by ultraviolet light (500 W), and the flocculation of P (AM/AA/AMPS) to dioctyl phthalate (DOP) in water was studied. However, compared with high-voltage ultraviolet light, low-pressure UV initiation has lower energy consumption, lower carbon environmental protection, and less heat produced in the reaction process [21]. The prepared polymer products have good stability, high purity, and short dissolution time.

In this present study, a flocculant PAMD was synthesized by AM, DMD and DPL under low-pressure UV initiation for flocculation of high-turbidity water. The optimum synthesis conditions are studied, including solid content, DPL content, DMD content, illumination time, and $\mathrm{pH}$. Flocculation performance of PAMD was evaluated in high-turbidity water purification, and the flocculation mechanism was discussed. The relationship between the factors and the experimental indexes was analyzed by the response surface method, and the optimal experimental conditions are determined for further application.

\section{Materials and Methods}

\subsection{Materials}

$\mathrm{AM}(99 \mathrm{wt} \%), \mathrm{DMD}(60 \mathrm{wt} \%)$, toluidine bule (TB, $2 \mathrm{~g} / \mathrm{L}$ aqueous solution), polyethylene potassium salt (PVSK, average Mw (162.21)n), and hexadecylpyridinium bromide (white powder, $96 \mathrm{wt} \%$ ) were all purchased from Shanghai Aladdin Industrial Corporation (Shanghai, China). DPL (solid content: $50 \mathrm{wt} \%$ ) was sourced from Huamai Biotechnology Co., Ltd. (Beijing, China). Photo-initiator 2-hydroxy-4'-(2-hydroxyethoxy)-2-methylpropiophenone (2959, $98 \mathrm{wt} \%$ ) was supplied by Changzhe Biological Technology Co., Ltd. (Shanghai, China). Kaolin (99 wt\%) without exception was obtained from Sinopharm Chemical Reagent Co., Ltd. (Beijing, China). In addition, hydrochloric acid ( $\mathrm{HCl}$, $\left.1.179 \mathrm{~g} / \mathrm{cm}^{3}\right)$ and sodium chloride $\left(\mathrm{NaOH}, 2.165 \mathrm{~g} / \mathrm{cm}^{3}\right)$ were obtained from Sinopharm Chemical Reagent Co., Ltd. for $\mathrm{pH}$ adjustment. AM was chemically pure, and the other reagents were analytical grade, which were used in this research without further purification.

\subsection{Preparation of $P A M D$}

Flocculant PAMD was copolymerized by AM, DMD, and DPL through low-pressure UV initiation in aqueous solution with 2959 as photo-initiator. In a transparent quartz jar, a certain amount of AM and DMD was totally dissolved in deionized water, and then a predetermined amount of DPL was slowly added. The mixture was stirred with a magnetic stirrer at $80 \mathrm{rpm}$ until complete mixing, and then the photo-initiator was added in the jar under pure nitrogen $\left(\mathrm{N}_{2}, 99.999 \%\right)$. After $20 \mathrm{~min}$, the quartz jar was sealed and transferred to a UV reaction device ( $48 \mathrm{~W}, 253.7 \mathrm{~nm}$, Gaojiang Scientific Co., 
Ltd., Suzhou, China) at room temperature for $2.5 \mathrm{~h}$. Thereafter, the flocculant PAMD was obtained and used in further experiments.

\subsection{Flocculation Experiments}

Half a gram of kaolin was prepared in $1 \mathrm{~L}$ of distilled water as synthetic wastewater. The $\mathrm{pH}$ of simulated wastewater was adjusted by $0.1 \mathrm{~mol} / \mathrm{L} \mathrm{HCl}$ or $0.1 \mathrm{~mol} / \mathrm{L} \mathrm{NaOH}$ aqueous solutions. Jar tests were conducted using $1 \mathrm{~L}$ jars in a stirring apparatus (Meiyu Instrument CO., Ltd., Wuhan, China) at ambient temperature, and the flocculation process was designed as follows: The kaolin-simulated wastewater was mixed after the addition of PAMD with a predetermined concentration under strong stirring (350 rpm) for $20 \mathrm{~min}$ and then slow stirring (50 rpm) for $15 \mathrm{~min}$. The supernatant (top $3 \mathrm{~cm}$ of water surface) was collected after a $30 \mathrm{~min}$ settling time using a syringe [22], and then measured through an ultraviolet-visible spectrophotometer with a wavelength of $550 \mathrm{~nm}$ (Beijing Purkinje General Instrument Co., Ltd., Beijing, China).

\section{Results and Discussion}

\subsection{OptimalSynthesis Conditions}

\subsubsection{Effect of Solid Content}

The solid content is the percentage of solid mass after removing volatile substances from organic matter and the total mass of organic matter. The difference in solid content indicates that the motion free degree of the various monomer molecules in the solution is various, and the results are displayed in Figure 1a. With the increase of solid content from $20 \%$ to $30 \%$, the intrinsic viscosity remarkably increased to $760 \mathrm{~mL} / \mathrm{g}$. However, the intrinsic viscosity decreased with the further increase of solid content. With cationic degree as an evaluation index, it could be seen that at low solid content, the cationic degree was relatively higher. With an increase in the organic matter content in the solution, the cationic degree suddenly dropped and then slowly increased. The possible reason may be that, at a low solid content, free radicals had free motion in dilute solutions and diffused to various parts to initiate polymerization along with the concentration gradient [23]. With an increase in solid content, the content of free radicals, AM, and cationic monomer contents would increase. When a large number of free radicals were exposed to AM, rapid polymerization occurs to produce a colloidal product to prevent free radical diffusion and reduce the flocculation property of the product [24]. Thus, $30 \%$ was selected as the optimum solid content in this experiment.

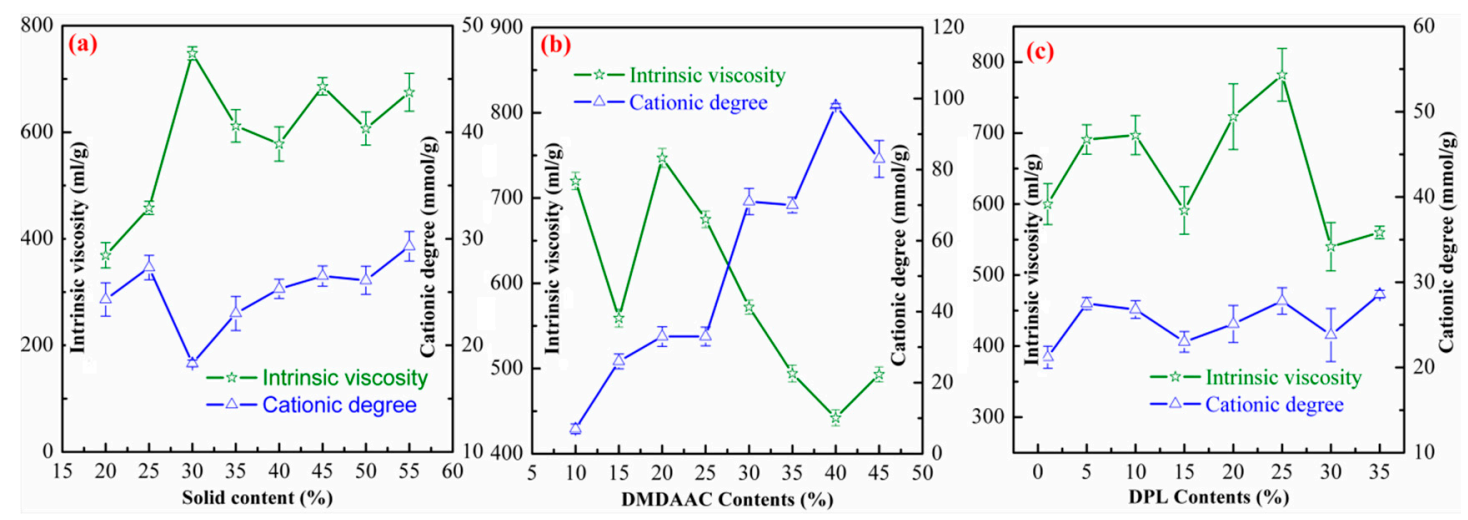

Figure 1. Effect of (a) solid content, (b) DMD content, and (c) DPL content on polymerization. DMD: dimethyl ammonium chloride; DPL: dodecyl polyglucoside.

\subsubsection{Effect of DMD Content}

In this study, AM was selected as the main chain, and DMD was used as a cationic monomer to participate in the polymerization. The content of the cationic monomer indicated the mass fraction 
of DMD mass and the total organic matter, which determined the size of the cationic degree of the synthetic product. As exhibited in Figure 1b, the mass fraction of cationic monomer increased from $10 \%$ to $40 \%$, and the cationic degree increased from $3.07 \mathrm{mmol} / \mathrm{g}$ to $58.7 \mathrm{mmol} / \mathrm{g}$. Then, with a further increase, the cationic degree began to decrease. When the mass fraction of DMD increased from $20 \%$ to $40 \%$, the intrinsic viscosity of the polymer prominently decreased with the increase in the cationic monomer content. This phenomenon was due to the different reactivity ratio of the AM monomer and DMD monomer in the polymerization. The reactivity ratio of the DMD monomer was obviously less than that of the AM monomer. As the content of the AM monomer was higher than that of the DMD monomer, the more intense the homo-polymerization of AM, the higher the intrinsic viscosity of the product. With the increase in the cationic monomer mass fraction, the AM content decreased, and the cationic degree of the product increased. However, due to the increase in the quaternary ammonium group, electrical mutual exclusion between each other and the generation of space hindrance, the viscosity of the product decreased [25]. Accordingly, as shown in Figure 1b, the growth trend of the intrinsic viscosity of the product was opposite to the cationic degree. In this study, $20 \%$ was determined to be the optimal DMD content.

\subsubsection{Effect of DPL Content}

In Figure 1c, the cationic degree remained unchanged at $25 \mathrm{mmol} / \mathrm{g}$ because the relative content of DMD and AM maintained unchanged in the reactor, while the cationic degree depended on the percentage of quaternary ammonium group in total molecular weight. With the increase of DPL mass fraction from 0 to $25 \%$, the intrinsic viscosity increased from $600 \mathrm{~mL} / \mathrm{g}$ to $760 \mathrm{~mL} / \mathrm{g}$, because DPL as a surfactant could ameliorate the surface of flocculants and promote the polymerization reaction. With the further increase in the DPL mass fraction, the DPL content in the reactor became too high, resulting in a decrease of the AM monomer content. At this time, the polymerization reaction was not as intense as that in the initial stage, and the solubility of the product decreased, causing a decrease in intrinsic viscosity [26]. Consequently, the optimal DPL content was 25\% for further use.

\subsubsection{Effect of Illumination Time}

The principle of polymerization of polymeric organic flocculants by UV light initiating polymerization of AM could be described by the classical free radical theory. In this study, under the action of $24 \mathrm{~W}$ low-pressure ultraviolet light, 2-hydroxy-4'-(2-hydroxyethoxy)-2methylpropiophenone(2959) was used as photo initiator to get the ability transition from the ground state to the excited state, engendering the free radical contacting AM monomer and opening the double bond to polymerize. Therefore, the UV illumination time had a vital effect on the polymerization. As displayed in Figure 2a, the intrinsic viscosity fluctuated in $600 \mathrm{~mL} / \mathrm{g}$ as the extension of the illumination time. With the increase of the illumination time from $60 \mathrm{~min}$ to $135 \mathrm{~min}$, the cationic degree of the product increased to $31 \mathrm{mmol} / \mathrm{g}$, and decreased as the illumination time extended further. At the early stage of illumination, UV irradiation on the surface of the solution, the rapid generation of substantial free radicals from the photo initiator initiated the synthesis with the AM molecules [27]. At this time, the polymerization reaction was intense, and the polymer organic matter was generated in large quantities. With the extension of the illumination time, an ivory-white gelatinous product was quickly synthesized at the bottom of the jar. As the ultraviolet light continued to radiate, the wave light was scattered and refracted in the place where the product had been synthesized, causing a rapid decrement of energy. It was difficult to ensure that the solution received enough energy to produce free radicals [28,29]. At this time, product synthesis depended on free radicals free movement that had been formed and a small number of free radicals produced autonomously. However, the parts that had been synthesized disproportionate with chain transfer to reduce the product performance because of the increase of light temperature over a long time. The results illustrated that low-pressure ultraviolet light could effectively induce polymerization, and the higher the synthesis efficiency, the more rapid the polymerization reaction. The effect of the intrinsic viscosity and cationic degree of the 
product on the flocculation performance was considered, and 135 min was selected as the optimal illumination time.

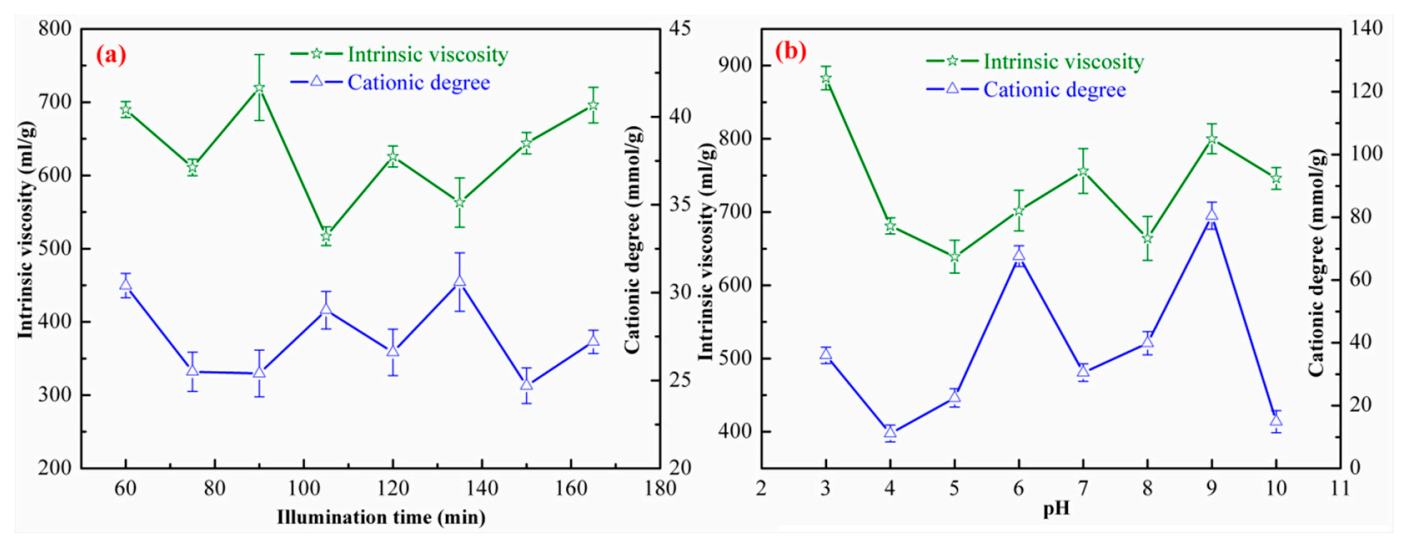

Figure 2. Effect of (a) illumination time and (b) $\mathrm{pH}$ on polymerization.

\subsubsection{Effect of $\mathrm{pH}$}

Using aqueous solution polymerization, the $\mathrm{pH}$ of aqueous solution not only affected the reaction rate, but also influenced the equilibrium of polymerization. In this experiment, $\mathrm{HCl}$ and $\mathrm{NaOH}$ were used to adjust the $\mathrm{pH}$ of the aqueous solution from 3 to 10, and the effects of diverse $\mathrm{pH}$ conditions on the intrinsic viscosity and cationic degree of the polymer were investigated in Figure $2 \mathrm{~b}$. Upon the increase of $\mathrm{pH}$ from 3 to 9 , the cationic degree of the polymer increased from $500 \mathrm{mmol} / \mathrm{g}$ to $700 \mathrm{mmol} / \mathrm{g}$; then the cationic degree decreased upon a further increase of $\mathrm{pH}$. With the increase of $\mathrm{pH}$ from 3 to 4 , the intrinsic viscosity dropped sharply, and fluctuated around $700 \mathrm{~mL} / \mathrm{g}$ as the increase of $\mathrm{pH}$. The reason was that, in acidic conditions, the amide group in AM was prone to an imidization reaction. Through a polymerization reaction it was easy to generate branched chains and the intrinsic viscosity of the product decreased [30,31]. Under alkaline conditions, methylation was prone to occur, accompanied by the hydrolysis of the quaternary ammonium group, and the intrinsic viscosity was unable to continue to rise. Accordingly, the optimal $\mathrm{pH}$ was deemed to be 9 .

\subsection{Flocculation Performance}

\subsubsection{Effect of DMD Content and Dosage}

The effect of DMD content (10-40\%) on the transmittance of supernatant was investigated, and the results are presented in Figure 3. The supernatant transmittance decreased with DMD content at the same dosage within $1.5-2.75 \mathrm{mg} / \mathrm{L}$. The supernatant transmittance exhibited higher stability during $96.5-97.9 \%$ as the DMD content was set at $20 \%$. However, the supernatant transmittance decreased remarkably as DMD content increased from $30 \%$ to $40 \%$. The results demonstrated that the higher the ratio of the cationic monomer to the total monomer mass, the shorter the length of the flocculant chain will be, resulting in a decrease in intrinsic viscosity. It was reported that a higher intrinsic viscosity proved favorable for adsorption and bridging between flocculants and pollutants in flocculation. Thus, the adsorption and bridging effect was significantly inhibited, leading to the deterioration of flocculation performance. Consequently, a DMD content of $20 \%$ was chosen in the following experiment. 


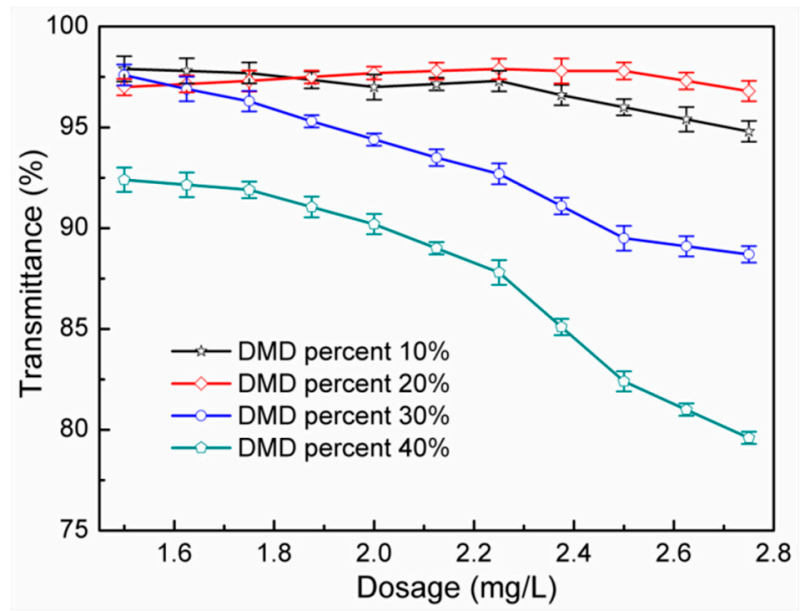

Figure 3. Effect of DMD dosage on transmittance.

\subsubsection{Effect of DPL Content and Dosage}

In order to improve the association and interaction between flocs in the flocculation process, the hydrophobic monomer DPL was introduced into PAMD. The effect of DPL content increase from $10 \%$ to $30 \%$ on the transmittance of the supernatant was investigated at various PAMD dosages, and the results are shown in Figure 4. The supernatant transmittance with varying DPL content reached more than $90 \%$, indicating that each sample had a good removal effect on the turbidity of the kaolin suspension. The supernatant transmittance at DPL content $20 \%$ was increased from $97 \%$ to $97.9 \%$ with a PAMD dosage of $1.5-2.25 \mathrm{mg} / \mathrm{L}$. However, with the increase in dosage, the supernatant transmittance at DPL content of $10 \%$ and $30 \%$ significantly decreased. The DPL content enlarging or decreasing were unsatisfactory for turbidity removal. Therefore, the optimal DPL content was considered to be $20 \%$ for further experiments.

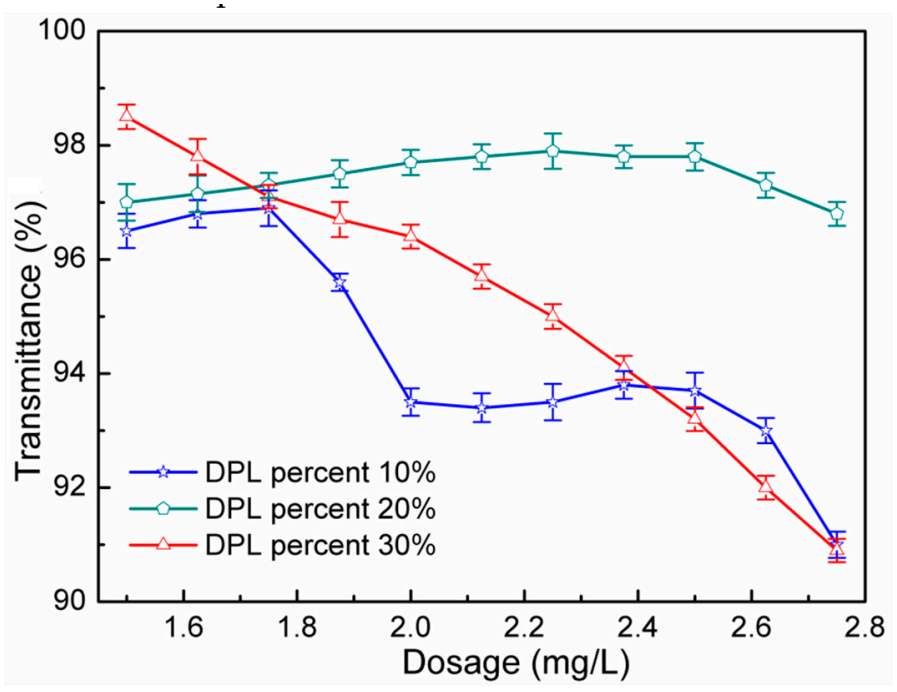

Figure 4. Effect of DPL dosage on transmittance.

\subsubsection{Effect of Deposition Time and $\mathrm{pH}$}

As displayed in Figure 5, the floc deposition time and $\mathrm{pH}$ were critical indexes to evaluate the flocculation performance. In Figure 5a, the transmittance of supernatant at PAMD dosage of $1.5 \mathrm{~g} / \mathrm{L}$ was significantly increased with the increase of deposition time in the range of 20-30 $\mathrm{min}$, and then kept stable at 30-40 $\mathrm{min}$. The results demonstrated that, because of the flocculation principle of flocculants to the kaolin particles in water, the large particles gathered gradually precipitate under the action of 
gravity, accompanied by sweeping effect. In addition, due to the small difference between gravity and buoyancy caused by small particles in water, the kaolin particles settled slowly after the deposition time of $30 \mathrm{~min}$. The change in hydraulic conditions results in a small amount of precipitated particles being dispersed in the water, and the supernatant transmittance has slightly decreased with a deposition time of $40-45 \mathrm{~min}$. It can be seen that the modified flocculant has good sedimentation performance and anti-shear ability, and $30 \mathrm{~min}$ was chosen as the optimum settling time in this experiment.
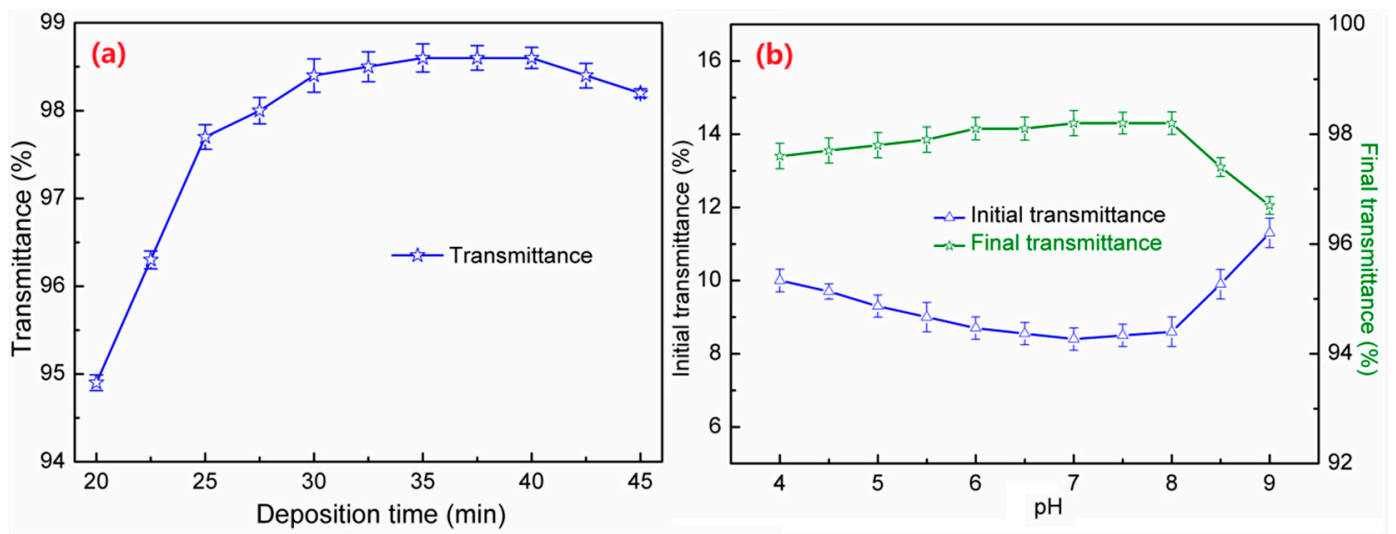

Figure 5. Effect of (a) deposition time and (b) $\mathrm{pH}$ on transmittance.

As exhibited in Figure 5b, the transmittance of raw water (turbidity: more than 1000 NTU) was $10 \%$ under strongly acidic conditions. The results showed that the siloxane substance in kaolin was hydrolyzed into negatively charged particles in water, which were encapsulated by a large number of $\mathrm{H}^{+}$ions under strongly acidic conditions. Thus, the transmittance of raw water was high. With the increase inpH from acidic to neutral, the water qualities become muddier and the transmittance was only $8.5 \%$. When the water quality was alkaline, the transmittance of the raw water increased to $11 \%$. In alkaline conditions, with the increase of hydroxyl ions in the water, the solubility of kaolin was inhibited and the water quality was turbid. After adding PAMD flocculant, the transmittance of the supernatant first increased and reached a maximum at $\mathrm{pH} 7$, and then decreased at $\mathrm{pH} 9$. The results demonstrated that the quaternary ammonium group dissociation of cationic organic flocculants was inhibited under acidic conditions, and the neutralization performance of flocculants could not be brought into full play, so the treatment effect was relatively poor. In alkaline conditions, a large number of free hydroxyl groups were dissociated in water, acting on quaternary ammonium groups, occupying the adsorption sites of organic flocculants to kaolin, and resulting in poor flocculation effect. This experiment not only obtained the optimum $\mathrm{pH}$, but also proved that the modified flocculant can adapt to the water quality of various $\mathrm{pH}$ conditions.

\subsubsection{Effect of Stirring Speed and Stirring Time}

In order to better mix the flocculant with the pollutants in the water, stirring is an essential step. The results are displayed in Figure 6. The fast stirring stage was the first step in the flocculation process, and determined the contact degree of the contaminants and the flocculant. As exhibited in Figure $6 a$, at different stirring speeds, the removal effect of flocculant on turbidity in water was good. With the increase in stirring speed, the removal effect was greatly improved. The reason was that with an increase of stirring speed, due to the more intense hydraulic agitation, pollutants frequently collide with organic flocculant macromolecules and more easily aggregate into large particles that can precipitate. With the stirring speed increased to $350 \mathrm{rpm}$, the fluctuation of water quality tended to be stable. However, under severe hydraulic conditions, the fragmentation and dispersion of flocs led to the decrease of collision probability between pollutants and flocculants. 

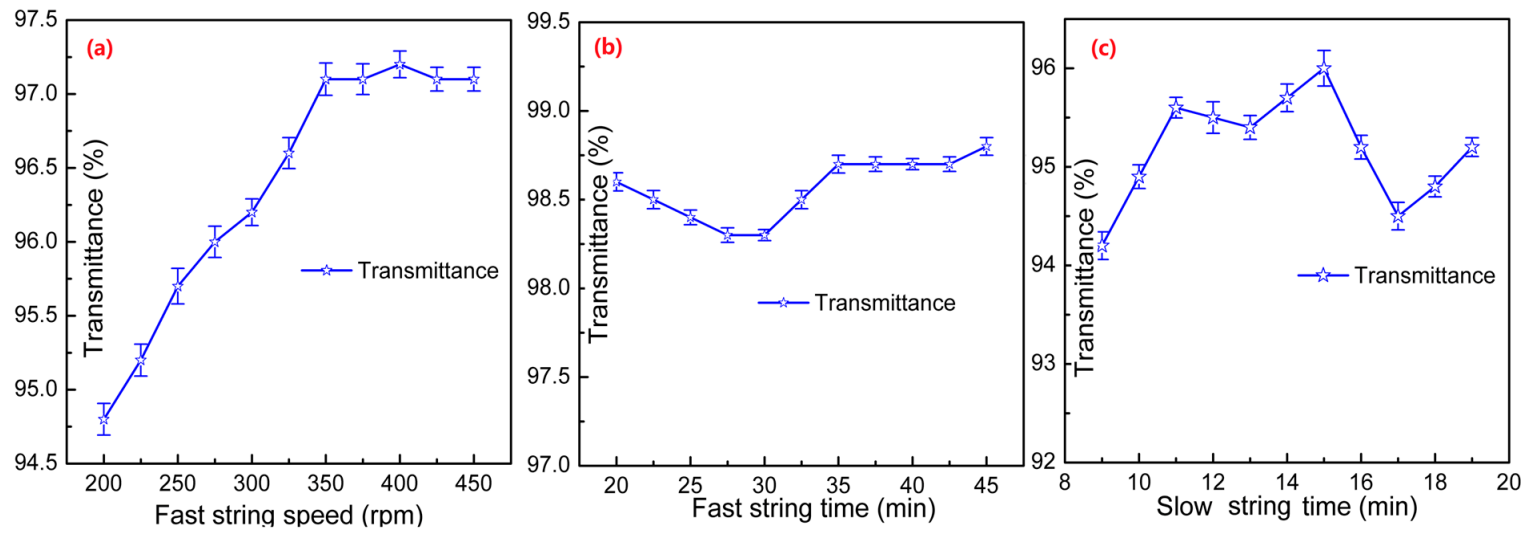

Figure 6. Effect of (a) fast stirring speed, (b) fast stirring time, and (c) slow stirring time on transmittance.

In Figure $6 b$, with the increase in stirring time, the change of flocculation effect was inconspicuous, illustrating that a fast stirring time had little influence on the experimental effect in this study. Under a suitably fast stirring speed, the probability of effective collision between flocculants and pollutants was greater. With the increase in stirring time, particle collision and stirring separation were synchronized to achieve dynamic stability equilibrium. At this time, the effect of stirring time on flocculation efficiency was very small. In this experiment, a better flocculation effect was achieved by making the stirring times all above $20 \mathrm{~min}$. The reason may be that, in the fast stirring stage, the organic flocculant dissolved in water was mainly in the form of long-chain organic molecules, which are uniform and difficult to disperse. Therefore, a longer stirring time was needed to achieve a better flocculation effect.

Slow stirring was designed to enable further contact of the combined particles to increase the weight of the floc and let it settle down. The results are exhibited in Figure 6c. With the increase in stirring time from $9 \mathrm{~min}$ to $15 \mathrm{~min}$, the transmittance of supernatant was increased from $94 \%$ to $96 \%$. The results illustrated that, with the increase in stirring time, the effective contact between the floc particles in the water made the flocs larger and the flocculation effect obviously improved. With the stirring time increased to over $15 \mathrm{~min}$, the flocs were broken under hydraulic action, splitting into small particle flocs or dispersing in water, and the flocculation effect was reduced. Accordingly, the appropriate slow stirring time was considered to be $15 \mathrm{~min}$.

\subsubsection{Comparison of Flocculation Performance between PAMD and PAC}

As shown in Figure 7, a comparison of the flocculation performance between PAMD and PAC was used to study the effect of low-pressure UV-induced polymerization. In Figure 7a, the transmittance of supernatant at PAMD significantly increased with increasing dosage before reaching maximum values at corresponding optimal ones. The supernatant transmittance reached $97 \%$ at a dosage of $1.5 \mathrm{mg} / \mathrm{L}$, and the corresponding turbidity was lower than $3 \mathrm{NTU}$. This is mainly the result of the combined action of electric neutralization and adsorption and bridging. In Figure $7 \mathrm{~b}$, the supernatant transmittance at PAC had an obvious change with the increase in dosage. When the dosage increased to $60 \mathrm{mg} / \mathrm{L}$, the transmittance reached a maximum of $98.8 \%$, and the turbidity of supernatant was lower than that of 2 NTU. The results showed that the flocculation effect of inorganic flocculant was slightly better than that of the organic flocculant, due to the high positive charge group of the inorganic flocculant. However, the PAC dosage required by the optimal condition was 30 times that of PAMD, which meant that high dosage, high energy consumption, and high cost were needed in practical application. In summary, the modified flocculant has wide application prospects. 

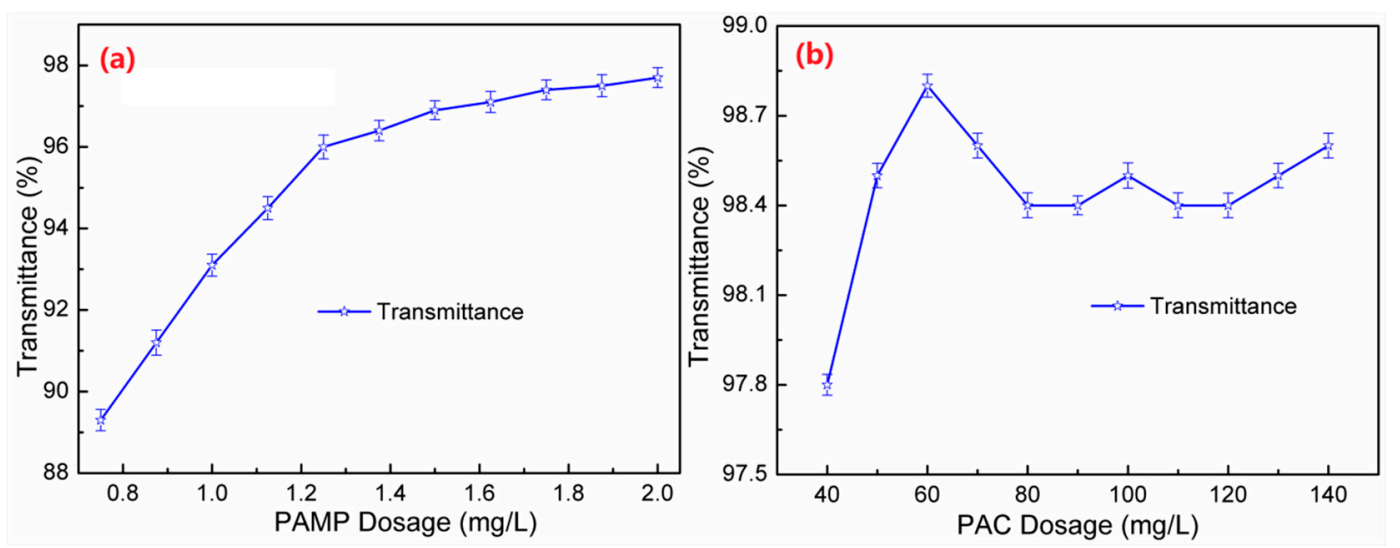

Figure 7. Effect of (a) PAMD dosage and (b) PAC dosage on transmittance. PAMD: polyacrylamide.

\subsection{Response Surface Method}

Response surface method was an experimental scheme design and optimization analysis based on statistical principles [32]. A mathematical model was established to explore the interaction between continuous variables, and the experimental conditions were more convenient than orthogonal experiments. In this experiment, the response surface method was used to explore the interaction effect of dosage (X1), fast stirring time (X2), $\mathrm{pH}(\mathrm{X} 3)$, and fast stirring speed (X4) on the flocculation effect. The transmittance of the supernatant was set as the target value, and the experimental design and analysis were carried out using Design Expert8.0 software (Informer Technologies Inc., https:/ /www.informer.com/).

As given in Figure 8a, at the same fast stirring time, the target value rose significantly with the increase of dosage from $2.0 \mathrm{mg} / \mathrm{L}$, and then increased slowly when the dosage was over $2.8 \mathrm{mg} / \mathrm{L}$. With the same dosage, the target value increased as the fast stirring time begun to increase from $15 \mathrm{~min}$, and presented the same trend as the preceding. The results show that the target value increased significantly under a low dosage and short, fast stirring time, which was because the flocculant needed to be uniformly contacted with pollutants in the water to achieve the desired result at a low dosage. Therefore, it is necessary to prolong the fast stirring time to give the flocculant adequate contact with the suspended substance.

Figure $8 \mathrm{~b}$ shows the interaction between dosage and $\mathrm{pH}$. The target value increased remarkably with the increase of dosage, and then increased slowly at the same $\mathrm{pH}$ conditions. At a dosage of $3.2 \mathrm{mg} / \mathrm{L}$, the change in the target value was slight, which was different from the results of the single-factor experiment. In strongly acidic or alkaline conditions, it was disadvantageous to the dissolution of flocculants and the accumulation of pollutants in water. The results demonstrated that there was a definite interaction between the dosage and $\mathrm{pH}$.

The interaction between the dosage and fast stirring speed was investigated in Figure $8 \mathrm{c}$. When the fast stirring speed was certain, the target value was proportional to the dosage, which was significant at low dosage and the change trend was faster. At a certain dosage, the target value increased with the increase in the stirring speed, and the target value was less affected when the stirring speed exceeded $300 \mathrm{rpm}$. In Figure 8c, the significant influence between dosage and the fast stirring speed was an ellipse, indicating that the interaction between the two was remarkable.

In Figure 9a, it can be seen from the shape of the ellipse that the interaction between stirring time and $\mathrm{pH}$ was obvious. At $\mathrm{pH} 7$, the target value increased with the stirring time from $10 \mathrm{~min}$ to $24 \mathrm{~min}$. The results indicated that the longer the stirring time, the more even the flocculant dispersing, which was more convenient for contacting and gathering with the pollutants. When the stirring time was certain, the transmittance had a slight change due to the increase in the $\mathrm{pH}$. The results were in agreement with the conclusions of the previous single-factor experiment. 

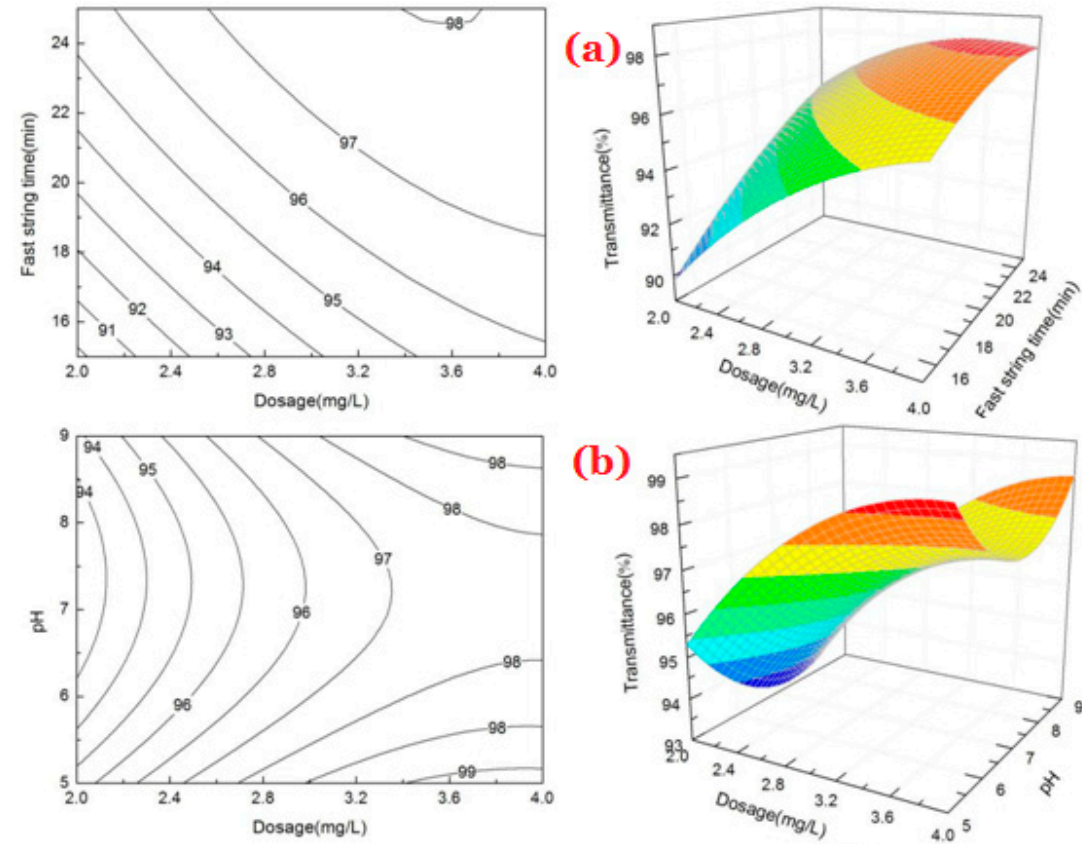

(b)
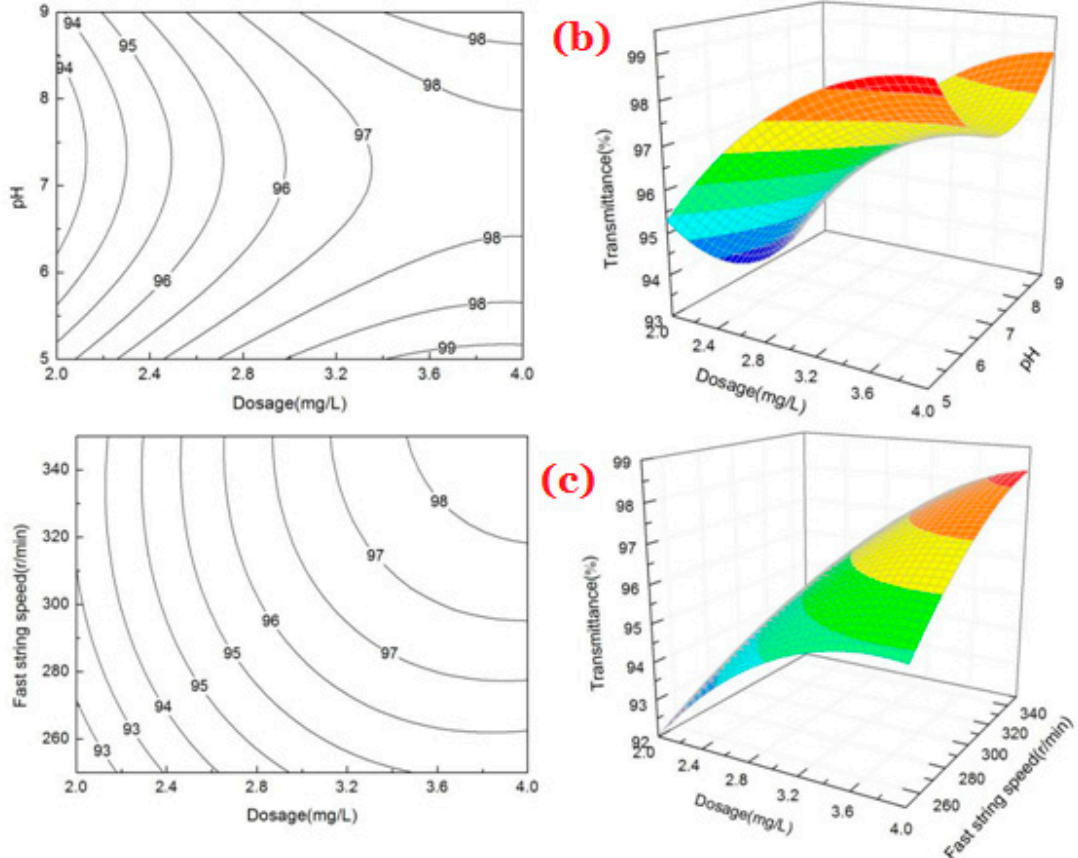

Figure 8. Response surface plotted and contour map of (a) dosage and fast stirring time, (b) dosage and $\mathrm{pH},(\mathbf{c})$ dosage and fast stirring speed.

The interaction between fast stirring time and fast stirring speed is shown in Figure $9 \mathrm{~b}$. At a certain fast stirring time, the target value decreased with the decrease in stirring speed, and the decrease speed was greatly accelerated. When the stirring speed was definite, the target value decreased with the decrease in stirring time and the minimum target value was $91.32 \%$ at 15 min of fast stirring and $250 \mathrm{r} / \mathrm{min}$ of stirring speed. The results indicated that at a low stirring speed and short stirring time, the inhibition of flocculation performance was caused by insufficient dissolving of the flocculant. Moreover, because of insufficient hydraulic action, the contaminant and flocculant failed to come into effective contact, resulting in poor flocculation.

Figure $9 \mathrm{c}$ shows the interaction between $\mathrm{pH}$ and fast stirring speed. Due to the effect of fast stirring speed and stirring time on hydraulic conditions, the effect on pollutants and flocculants in water was the same, and the interaction effect was not significant. The experimental results showed that the optimal $\mathrm{pH}$ for turbidity removal was neutral, as strong acidity or strong alkalinity inhibited the effect of the flocculant. Under strongly acidic or alkaline conditions, the target value decreased with the decrease in stirring speed. The minimum value of the experimental supernatant was $95 \%$ at stirring time $250 \mathrm{r} / \mathrm{min}$ and $\mathrm{pH} 6.5$ or 8.5. A single-factor experiment showed that the initial permeability was relatively high under strongly acidic and alkaline conditions, so the removal efficiency was not as good as in neutral conditions. 

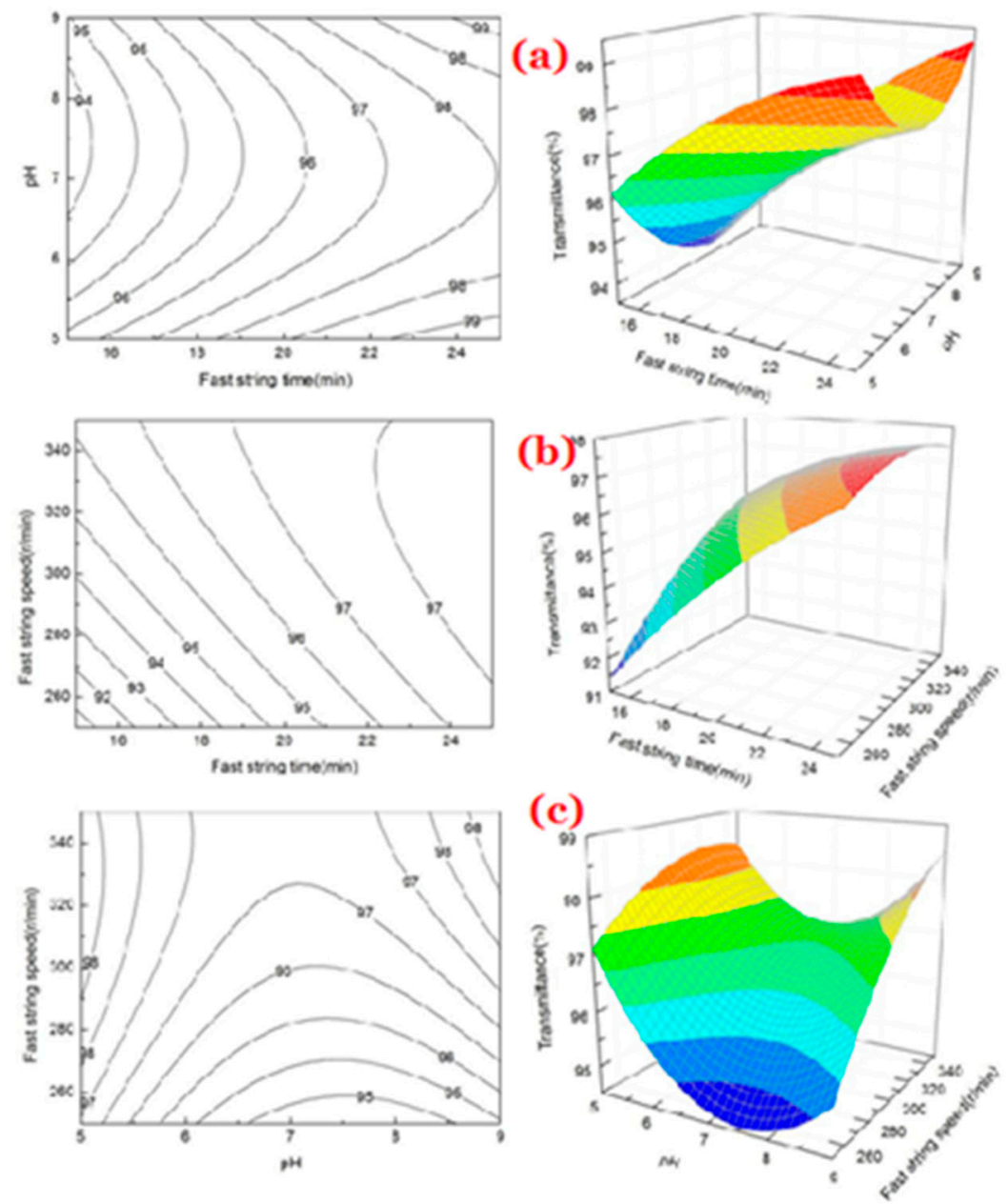

Figure 9. Response surface plotted and contour map of (a) fast stirring time and $\mathrm{pH}$, (b) fast stirring time and fast stirring speed, and (c) $\mathrm{pH}$ and fast stirring speed.

\subsection{Turbidity Removal Mechanism}

The removal effect of modified flocculant on the turbidity of water was obtained through a series of experiments, and the optimal experimental condition was dosage $4 \mathrm{mg} / \mathrm{L}$, fast stirring time $20 \mathrm{~min}$, $\mathrm{pH} 7$, and fast stirring speed $320 \mathrm{rpm}$. The principle of flocculant removal of turbidity was analyzed for further study of the performance of flocculants. The Fourier transform-infrared (FTIR) spectra of PAMD, kaolin particles, and flocs are exhibited in Figure 10. The peaks at $800 \mathrm{~cm}^{-1}$ and $1097 \mathrm{~cm}^{-1}$ were ascribed to $\mathrm{Si}-\mathrm{O}$ and $\mathrm{Si}-\mathrm{O}-\mathrm{Si}$, respectively. The results indicate that the main components of kaolin were compounds of Si and O [33]. The existence of two peaks on the spectrum of kaolin and floc indicated that no chemical reaction occurred in the coagulation process due to the absence of a new group. The absorption peaks at $3412 \mathrm{~cm}^{-1}$ and $1664 \mathrm{~cm}^{-1}$ were attributed to the stretching vibration of $-\mathrm{NH}_{2}$ and the stretching of $\mathrm{C}=\mathrm{O}$ in amide groups, respectively [34,35]. However, the two peaks were non-existent in the spectra of flocs, because the PAMD dosage was lower than the content of kaolin particles in water.

As presented in Figure 11, a schematic diagram of the removal of kaolin from water by the modified flocculant was conjectured based on the analysis of the infrared spectrum of kaolin, floc, and PAMP, and the $\mathrm{pH}$ experiment. Under acidic and neutral conditions, the quaternary ammonium group of PAMD was electrolyzed to $\mathrm{NH}_{4}{ }^{+}$, which produced electric adsorption on free negative charged kaolin and formed floc particles. These floc particles were gathered together to form large reticulate particles through the effect of bridging and hydrophobic association under continuous agitation. Due to the existence of the hydrophobic group and the settlement of its own gravity, the particles in 
water were further aggregated through the sweeping effect to achieve turbidity removal. The cationic electrical adsorption capacity of the quaternary ammonium group was inhibited due to the increase of hydroxyl ions in the water under alkaline conditions. In summary, the adsorption of the long-chain molecule of flocculant played a dominant role in flocculation, and the hydrophobic association also played an important role in flocculation. Finally, the remaining pollutants in the water were further removed under the sweeping effect.

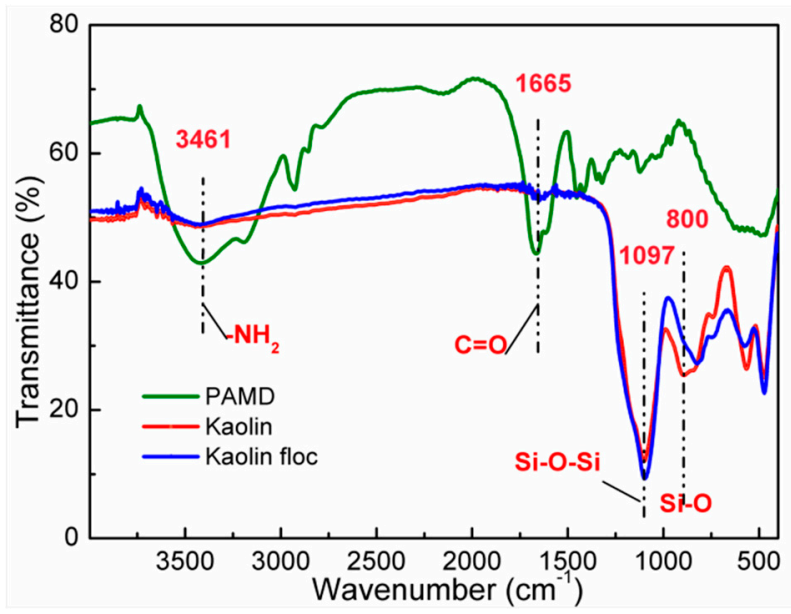

Figure 10. FTIR comparison of PAMD, kaolin, and flocs. FTIR: Fourier transform-infrared.

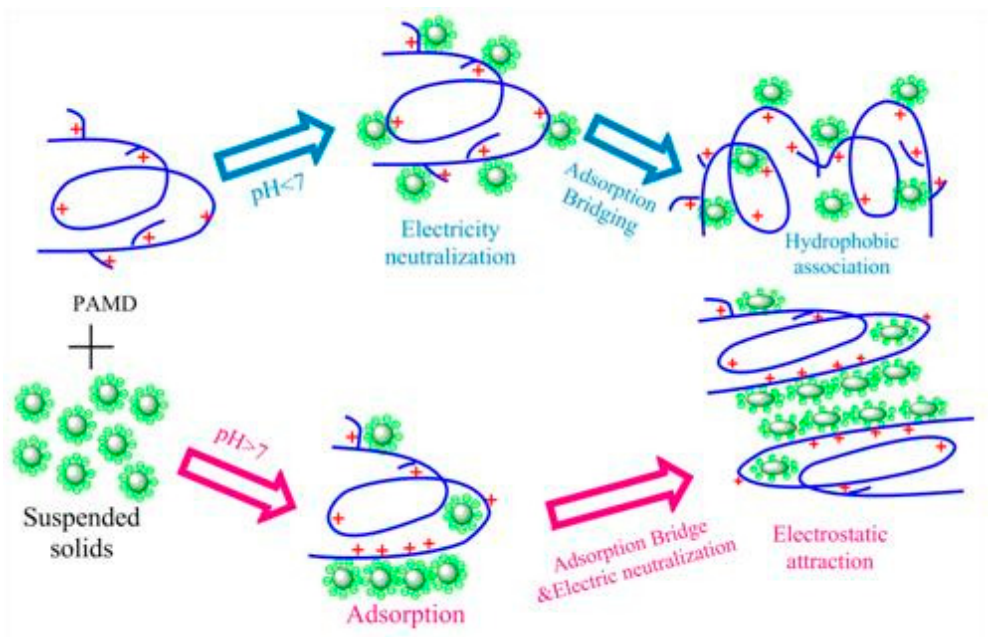

Figure 11. Flocculation mechanisms for PAMD.

\section{Conclusions}

In this study, a hydrophobic cationic polyacrylamide flocculant was synthesized through low-pressure UV initiated copolymerization. Key influencing factors, such as solid content, monomer concentration, illumination time, and $\mathrm{pH}$, were systemically investigated based on intrinsic viscosity, cationic degree, and supernatant transmittance. The following optimum synthesis conditions were obtained: solid content of $30 \%$, DPL content of $25 \%$, DMD content of $30 \%$, illumination time of $135 \mathrm{~min}$, and $\mathrm{pH}$ of 9. Experimental simulation results of response surface method illustrated that the optimum experimental conditions were as follows: dosage of $4 \mathrm{mg} / \mathrm{L}$, fast stirring time of $20 \mathrm{~min}, \mathrm{pH}$ of 7 , stirring speed of $320 \mathrm{r} / \mathrm{min}$, and transmittance of supernatant $98.9 \%$. The flocculation performance of high-turbidity water was investigated and compared with that of PAC. Experimental results demonstrated that the optimum flocculation efficiency was observed at a dosage of $2.0-2.8 \mathrm{mg} / \mathrm{L}$, deposition time of 25-35 min, $\mathrm{pH}$ of 6-8, fast stirring speed of 300-400 rpm, slow stirring time 13-16 $\mathrm{min}$, and fast stirring time of $20-35 \mathrm{~min}$ with $97.5-98.7 \%$ supernatant transmittance. Comparative 
experimental results indicated that PAMD has a broader prospect of application than PAC. Therefore, PAMD could be applied as a highly efficient and environmentally friendly flocculant in high-turbidity water treatment.

Author Contributions: J.M. (Jiangya Ma) conceived and designed the experimental contents and scheme; X.F. (Xue Fu) and W.X. (Wei Xia) performed the flocculation experiments; K.F. (Kun Fu) analyzed the data; Y.L. (Yi Liao) and the other authors contributed to paper writing.

Funding: The study was financially supported by the National Nature Science Foundation of China (Project Nos. 51878001, 51408004), the University Natural Science Research Key Project of Anhui Province (Project No. KJ2017A061), and the Department of Science and Technology of Sichuan Province (Project No. 2017JY0129).

Conflicts of Interest: The authors declare no conflict of interest.

\section{References}

1. Knaeps, E.; Ruddick, K.G.; Doxaran, D.; Dogliotti, A.I.; Nechad, B.; Raymaekers, D.; Sterckx, S. A SWIR based algorithm to retrieve total suspended matter in extremely turbid waters. Remote Sens. Environ. 2015, 168, 66-70. [CrossRef]

2. Mores, R.; Treichel, H.; Zakrzevski, C.A.; Kunz, A.; Steffens, J.; Dallago, R.M. Remove of phosphorous and turbidity of swine wastewater using electro coagulation under continuous flow. Sep. Purif. Technol. 2016, 171, 112-115. [CrossRef]

3. Zahrim, A.Y.; Tizaoui, C.; Hilal, N. Coagulation with polymers for nano filtration pre-treatment of highly concentrated dyes: A review. Desalination 2011, 266, 1-5. [CrossRef]

4. Crini, G.; Badot, P.M. Application of chitosan, a natural aminopolysaccharide, for dye removal from aqueous solutions by adsorption processes using batch studies: A review of recent literature. Prog. Polym. Sci. 2008, 33, 399-447. [CrossRef]

5. Arena, F.; Chio, R.D.; Gumina, B.; Spadaro, L.; Trunfio, G. Recent advances on wet air oxidation catalysts for treatment of industrial wastewaters. Inorg. Chim. Acta 2015, 431, 101-109. [CrossRef]

6. Guan, Q.Q.; Zhu, G.C.; Liao, Y.; Xu, J.; Sun, X.X.; Tian, F.; Xu, J.X.; Luo, M.H. Preparation, characterization, and sludge conditioning of cationic polyacrylamide synthesized by a novel UVA-initiated system. Processes 2018, 6, 233. [CrossRef]

7. Cai, T.; Li, H.J.; Yang, R.; Wang, Y.W. Efficient flocculation of an anionic dye from aqueous solutions using a cellulose-based flocculant. Cellulose 2015, 22, 1439-1449. [CrossRef]

8. Ma, J.Y.; Shi, J.; Ding, H.C.; Zhu, G.C.; Fu, K.; Fu, X. Synthesis of cationic polyacrylamide by low-pressure UV initiation for turbidity water flocculation. Chem. Eng. J. 2017, 312, 20-29. [CrossRef]

9. Huang, M.; Wang, Y.W.; Cai, J.; Bai, J.F.; Yang, H.; Li, A.M. Preparation of dual-function starch-based flocculants for the simultaneous removal of turbidity and inhibition of $E$. coli in water. Water Res. 2016, 98, 128-137. [CrossRef]

10. Bolto, B.; Gregory, J. Organic polyelectrolytes in water treatment. Water Res. 2007, 41, 2301-2324. [CrossRef]

11. Lapointe, M.; Barbeau, B. Substituting polyacrylamide with an activated starch polymer during ballasted flocculation. J. Water Process Eng. 2019, 28, 129-134. [CrossRef]

12. Kumar, K.; Adhikary, P.; Karmakar, N.C.; Gupta, S.; Singh, R.P.; Krishnamoorthi, S. Synthesis, characterization and application of novel cationic and amphoteric flocculants based on amylopectin. Carbohydr. Polym. 2015, 127, 275-281. [CrossRef] [PubMed]

13. Rao, N.R.H.; Granville, A.M.; Browne, C.; Dagastine, R.R.; Yap, R.; Jefferson, B.; Henderson, R.K. Determining how polymer-bubble interactions impact algal separation using the novel "Posi"-dissolved air flotation process. Sep. Purif. Technol. 2018, 201, 139-147.

14. Liu, F.; Ma, B.R.; Zhou, D.; Zhu, L.J.; Fu, Y.Y.; Xue, L.X. Positively charged loose nanofiltration membrane grafted by diallyl dimethyl ammonium chloride (DADMAC) via UV for salt and dye removal. React. Funct. Polym. 2015, 86, 191-198. [CrossRef]

15. Lv, S.H.; Sun, T.; Zhou, Q.F.; Liu, J.J.; Ding, H.D. Synthesis of starch-g-p(DMDAAC) using HRP initiation and the correlation of its structure and sludge dewater ability. Carbohydr. Polym. 2014, 103, 285-293. [CrossRef] [PubMed]

16. Yang, Z.L.; Gao, B.Y.; Li, C.X.; Yue, Q.Y.; Liu, B. Synthesis and characterization of hydrophobically associating cationic polyacrylamide. Chem. Eng. J. 2010, 161, 27-33. [CrossRef] 
17. Wang, L.J.; Wang, J.P.; Yuan, S.J.; Zhang, S.J.; Tang, Y.; Yu, H.Q. Gamma radiation-induced dispersion polymerization in aqueous salts solution for manufacturing a cationic flocculant. Chem. Eng. J. 2009, 149, 118-122. [CrossRef]

18. Mishra, S.; Mukul, A.; Sen, G.; Jha, U. Microwave assisted synthesis of polyacrylamide grafted starch (St-g-PAM) and its applicability as flocculant for water treatment. Int. J. Biol. Macromol. 2011, 48, 106-111. [CrossRef]

19. Guan, Q.Q.; Zheng, H.L.; Zhai, J.; Zhao, C.; Zheng, X.K.; Tang, X.M.; Chen, W.; Sun, Y.J. Effect of template on structure and properties of cationic polyacrylamide: Characterization and mechanism. Ind. Eng. Chem. Res. 2014, 53, 5624-5635. [CrossRef]

20. Zheng, H.L.; Ma, J.Y.; Zhu, C.J.; Zhang, Z.; Liu, L.W.; Sun, Y.J.; Tang, X.M. Synthesis of anion polyacrylamide under UV initiation and its application in removing dioctyl phthalate from water through flocculation process. Sep. Purif. Technol. 2014, 123, 35-44. [CrossRef]

21. Ma, J.Y.; Fu, K.; Jiang, L.Y.; Ding, L.; Guan, Q.Q.; Zhang, S.H.; Zhang, H.W.; Shi, J.; Fu, X. Flocculation performance of cationic polyacrylamide with high cationic degree in humic acid synthetic water treatment and effect of kaolin particles. Sep. Purif. Technol. 2017, 181, 201-212. [CrossRef]

22. Andreoli, F.C.; Sabogal-paz, L.P. Coagulation, flocculation, dissolved air flotation and filtration in the removal of Giardia spp. and Cryptosporidium spp. from water supply. Environ. Technol. 2019, 40, 654-663. [CrossRef] [PubMed]

23. Chen, L.; Zhu, H.; Sun, Y.H.; Chiang, P.C.; Sun, W.Q.; Xu, Y.H.; Zheng, H.L.; Shah, K.J. Characterization and sludge dewatering performance evaluation of the photo-initiated cationic flocculant PDD. J. Taiwan Inst. Chem. Eng. 2018, 93, 253-262. [CrossRef]

24. Sun, Q.; Zheng, H.L.; Zheng, X.Y.; Hu, X.B.; An, Y.Y.; Liu, H.X.; Zhao, C.L. Dual polydopamine-anion polyacrylamide polymer system for improved removal of nickel ions and methylene blue from aqueous solution. Sci. Adv. Mater. 2019, 11, 116-127. [CrossRef]

25. Schwarz, D.; Weber, J. Synthesis of mesoporouspoly(melamine-formaldehyde) particles by inverse emulsion polymerization. J. Colloid Interface Sci. 2017, 498, 335-342. [CrossRef] [PubMed]

26. Zhao, C.L.; Zheng, H.L.; Gao, B.Y.; Liu, B.Z.; Zhai, J.; Zhang, S.X.; Xu, B.C. Ultrasound-initiated synthesis of cationic polyacrylamide for oily wastewater treatment: Enhanced interaction between the flocculant and contaminants. Ultrason. Sonochem. 2018, 42, 31-41. [CrossRef]

27. Wang, S.; Hou, Q.; Kong, F.; Fatehi, P. Production of cationic xylan-METAC copolymer as a flocculant for textile industry. Carbohydr. Polym. 2015, 124, 229-236. [CrossRef]

28. Liao, Y.; Zheng, H.L.; Qian, L.; Sun, Y.J.; Dai, L.; Xue, W.W. Uv-initiated polymerization of hydrophobically associating cationic polyacrylamide modified by a surface-active monomer: A comparative study of synthesis, characterization, and sludge dewatering performance. Ind. Eng. Chem. Res. 2014, 53, 11193-11203. [CrossRef]

29. Shaban, M.; S.A., A.R.; Ahadian, M.M.; Tamsilian, Y.; Weber, A.P. Facile synthesis of cauliflower-like hydrophobically modified polyacrylamide nanospheres by aerosol-photopolymerization. Eur. Polym. J. 2016, 83, 323-336. [CrossRef]

30. Alange, V.V.; Birajdar, R.P.; Kulkarni, R.V. Functionally modified polyacrylamide-graft-gum karaya $\mathrm{pH}$-sensitive spray dried microspheres for colon targeting of an anti-cancer drug. Int. J. Biol. Macromol. 2017, 102, 829-839. [CrossRef]

31. Thapa, K.B.; Qi, Y.; Clayton, S.A.; Hoadley, A.F. Lignite aided dewatering of digested sewage sludge. Water Res. 2009, 43, 623-634. [CrossRef] [PubMed]

32. Yang, Z.H.; Huang, J.; Zeng, G.M.; Ruan, M.; Zhou, C.S.; Li, L.; Rong, Z.G. Optimization of flocculation conditions for kaolin suspension using the composite flocculant of MBFGA1 and PAC by response surface methodology. Bioresour. Technol. 2009, 100, 4233-4239. [CrossRef] [PubMed]

33. Worasith, N.; Goodman, B.A.; Neampan, J.; Jeyachoke, N.; Thiravetyan, P. Characterization of modified kaolin from the Ranong deposit Thailand by XRD, XRF, SEM, FTIR and EPR techniques. Clay Miner. 2011, 46, 539-543. [CrossRef] 
34. Yang, Z.; Yan, H.; Yang, H.; Li, H.B.; Li, A.M.; Cheng, R.S. Flocculation performance and mechanism of graphene oxide for removal of various contaminants from water. Water Res. 2013, 47, 3037-3046. [CrossRef] [PubMed]

35. Manafi, M.R.; Manafi, P.; Agarwal, S.; Bharti, A.K.; Asif, M.; Gupta, V.K. Synthesis of nanocomposites from polyacrylamide and graphene oxide: Application as flocculants for water purification. J. Colloid Interface Sci. 2017, 490, 505-510. [CrossRef] 